(6) OPEN ACCESS

\title{
High-risk periodontal pathogens contribute to the pathogenesis of atherosclerosis
}

\author{
Bradley Field Bale, ${ }^{1}$ Amy Lynn Doneen, ${ }^{1}$ David John Vigerust ${ }^{2}$
}

'Texas Tech Health Science Center, School of Nursing, Lubbock, Texas, USA 2Department of Neurological Surgery, Vanderbilt University School of Medicine, Nashville, Tennessee, USA

Correspondence to Dr Bradley Field Bale, 1002 Montrose Dr, Gallatin, TN 37066, USA;

bbale@baledoneen.com

Received 7 June 2016 Revised 1 November 2016 Accepted 5 November 2016 Published Online First 29 November 2016

\section{ABSTRACT}

Periodontal disease (PD) is generated by microorganisms. These microbes can enter the general circulation causing a bacteraemia. The result can be adverse systemic effects, which could promote conditions such as cardiovascular disease. Level A evidence supports that PD is independently associated with arterial disease. PD is a common chronic condition affecting the majority of Americans 30 years of age and older. Atherosclerosis remains the largest cause of death and disability. Studies indicate that the adverse cardiovascular effects from PD are due to a few putative or high-risk bacteria: Aggregatibacter actinomycetemcomitans, Porphyromonas gingivalis, Tannerella forsythia, Treponema denticola or Fusobacterium nucleatum. There are three accepted essential elements in the pathogenesis of atherosclerosis: lipoprotein serum concentration, endothelial permeability and binding of lipoproteins in the arterial intima. There is scientific evidence that PD caused by the high-risk pathogens can influence the pathogenesis triad in an adverse manner. With this appreciation, it is reasonable to state PD, due to high-risk pathogens, is a contributory cause of atherosclerosis. Distinguishing this type of PD as causal provides a significant opportunity to reduce arterial disease.

\section{BACKGROUND}

Bacteraemia with germs from the oral cavity was well documented in a publication in 1954. The landmark study indicated systemic spread of oral microbes occurs frequently. The per cent incidence found was $40 \%$ with periodontal cleaning, 35\% with dental extractions, 24\% with brushing and up to $17 \%$ with mastication. ${ }^{1}$ The spread of these oral bacteria throughout the body happens quickly. This results in acute and chronic inflammation, which can be pathological. High-risk periodontal pathogens include Aggregatibacter actinomycetemcomitans (Aa), Porphyromonas gingivalis (Pg), Tannerella forsythia (Tf), Treponema denticola (Td) and Fusobacterium nucleatum (Fn). They are prevalent in periodontitis. These germs enter the systemic circulation directly, and they also produce endotoxins such as lipopolysaccharides (LPS). These endotoxins generate inflammatory cytokines, upregulate endothelial adhesion molecules and induce a prothrombotic environment. These actions can favour the formation of arterial disease and can enhance the risk of an atherothrombotic event. ${ }^{2}$ Using DNA to identify putative oral bacteria, several studies have documented their presence within atheroma. In 2009, 44 patients underwent coronary endarterectomies. Thirty-nine of the subjects had periodontal disease (PD). Thirty-six of the specimens from patients with PD were positive for oral pathogens.
The most common were $P g$ and $A a$. Sixty-four per cent of those atheromas had two or more pathogens. Only one of the atheroma from a patient without PD demonstrated any oral pathogens. ${ }^{3}$ In 2011, 42 carotid endarterectomy specimens were analysed for oral pathogen DNA. Every atheroma had at least one pathogen, and many had multiple pathogens. Again, the most common bacteria were $\mathrm{Pg}$ and $\mathrm{Aa} .{ }^{4}$ Oral pathogens create bacteraemia, and those bacteria, especially the high-risk microbes, are frequently associated with atherosclerotic lesions.

The American Heart Association (AHA) stated after an extensive review of the literature that PD was independently associated with arteriosclerotic vascular disease (ASVD). This relationship was demonstrated with level A evidence. They discussed in their statement several plausible mechanisms by which PD could be associated with arterial disease. One explanation involves systemic inflammation, which can occur with periodontitis. This has been documented by increased levels of biomarkers such as high-sensitivity C-reactive protein, tumour necrosis factor- $\alpha$ (TNF- $\alpha$ ) and interleukin 6. PD has been associated with the stimulation of the innate immune system via toll-like receptors (TLRs). TLRs can trigger the activation of nuclear factor $\kappa \beta(\mathrm{NF}-\kappa \mathrm{B})$, which can create increased levels of adhesion molecules stimulating endothelial dysfunction as well as increased inflammatory cytokines. The possibility of LPSs and heat-shock proteins coming from the PD pathogens causing an autoimmune-type reaction from $\mathrm{T}$ and $\mathrm{B}$ cells was mentioned. Another potential mechanism discussed was direct arterial damage from the bacteria in the blood stream. They acknowledged that there are studies showing PD therapy has improved biomarkers of systemic inflammation and even surrogate indicators of subclinical arterial disease. However, due to the fact that there is no definite evidence to support the claim that treating PD decreases cardiovascular $(\mathrm{CV})$ events, the AHA went on to comment that PD could not be considered causal of ASVD. ${ }^{5}$ From a clinical perspective, there is a significant difference between being associated with versus being causal of a disease. Optimal management of an associated condition may not impact the end disease, whereas such management of a causal condition would have a favourable effect on the end disease. Since the AHA generated their statement about the relationship of PD to ASVD, new evidence has emerged which argues PD caused by the high-risk pathogens, $\mathrm{Aa}, \mathrm{Pg}, \mathrm{Tf}, \mathrm{Td}$ or $\mathrm{Fn}$, can enhance elements of the atherosclerosis pathogenesis triad. Due to this scientific knowledge, PD caused by these putative bacteria can be considered causal of ASVD. This is a significant clinical 
distinction, which means PD due to these organisms should be optimally treated to mitigate the risk of ASVD.

Recent publications indicate that the formation of atherosclerosis is dependent upon three essentials: serum lipoprotein concentration, endothelial permeability and lipoprotein binding in the intima. ${ }^{6}$ If a medical condition can influence all of these critical elements in a manner favouring the pathogenesis of atherosclerosis, it can be concluded to be a contributory cause of ASVD. A contributory cause does not require that all those who possess the contributory cause experience the disease, nor does it require that all those who are free of the contributory cause be free of the disease. It also means the contributory cause may not be necessary to experience the disease. ${ }^{7} \mathrm{PD}$ is independently associated with ASVD; substantial evidence and mechanistic understanding now exist that strongly suggest PD, due to the above high-risk pathogens, is a contributory cause of ASVD. Herein, we present a summary of this evidence and understanding. Based on these findings, PD resulting from high-risk pathogens should be considered causal of ASVD.

\section{PATHOGENESIS, PART 1: SERUM LIPOPROTEIN CONCENTRATION}

Atherogenic lipoprotein particles are heterogeneous, containing various amounts of cholesterol. Each particle has an apolipoprotein $\mathrm{B}(\mathrm{ApoB})$ on its surface. Wilkins et $a l^{6}$ recently demonstrated that ApoB concentration in the serum is more predictive of the pathogenesis of ASVD than serum cholesterol concentration. In addition to this study, previous studies have also shown the best lipoprotein predictor of cardiovascular disease risk is ApoB ${ }^{8-10}$ There is a direct positive and independent association with ApoB concentration and small, dense low-density lipoprotein (sd-LDL) concentrations. ${ }^{11}$ Therefore, an essential element in the pathogenesis of ASVD, namely ApoB concentration, is increased with elevating levels of sd-LDL. Secretory phospholipase A2 (sPLA2) modifies LDL into sd-LDL. ${ }^{12}$ The activity of sPLA2 has been shown to increase in subjects with high-risk periodontal pathogens $\mathrm{Aa}, \mathrm{Pg}, \mathrm{Tf}$ and $\mathrm{Td}$. The higher the burden of these pathogens, the greater the activity of sPLA2. ${ }^{13}$ Generalised aggressive PD is associated with the putative pathogens $P g$ and $A a \cdot{ }^{14}$ It is demonstrated that patients with generalised aggressive periodontitis have twice the concentration of sd-LDL compared with subjects without PD. ${ }^{15}$ This essentially means the concentration of ApoB is doubled. PD due to highrisk pathogens can increase the plasma concentration of ApoB, which will promote the pathogenesis of ASVD.

\section{PATHOGENESIS, PART 2: ENDOTHELIAL PERMEABILITY}

Formation of an atheroma requires monocytes along with lipoproteins to penetrate the endothelium. It is the accumulation of lipoproteins in the intima that creates the core of the atheromatous lesion. The permeability of the endothelium is a critical factor in the pathogenesis of ASVD. ${ }^{16}$ Dysfunctional endothelium becomes more permeable. It is proposed that endothelial dysfunction is the initial biological malady leading to ASVD. ${ }^{17}$ PD can generate endothelial dysfunction by several mechanisms. The high-risk gram-negative pathogens $(\mathrm{Aa}, \mathrm{Pg}, \mathrm{Tf}, \mathrm{Td})$ generate LPSs, which trigger the innate immune system. LPSs stimulate TLRs, which are ubiquitous and present in endothelial cells. TLR signalling activates the genetic transcription factor NF- $\kappa$ B. This in turn leads to elevated levels of endothelial cellular adhesion molecules and TNF- $\alpha{ }^{18}$ Cellular adhesion molecules capture monocytes and increase the permeability of the endothelium. ${ }^{19}$ TNF- $\alpha$ is known to induce increased endothelial permeability via its effect on tight junction proteins. ${ }^{20}$ Chronic periodontal infections with high-risk pathogens can lead to increased permeability of the endothelium via the innate immune system.

Several other mechanisms exist by which PD can generate dysfunctional and permeable endothelium. Endothelial integrity depends on its endogenous capacity for repair. Persistent exposure to inflammation leads to senescence of endothelial cells and their detachment, with consequent increased permeability. This adverse injury can be repaired by replication of adjacent endothelial cells or by formation of new mature endothelial cells from endothelial progenitor cells. ${ }^{21}$ The high-risk PD pathogen (Aa) produces a toxic protein known as leukotoxin (LtxA). This toxin has been shown to generate endothelial apoptosis along with impaired proliferation and increase in endothelial adhesion molecules. $^{22}$ These adverse effects of LtxA will lead to increased penetrability of the endothelium.

PD can impair the structural coupling of endothelial cells. Cadherin proteins lock endothelial cells tightly together. Any disruption of endothelial cadherin will result in endothelial dysfunction and enhanced permeability. ${ }^{23}$ The PD pathogen Fn possesses a surface adhesion molecule called FadA. There are two forms of FadA. One is a non-secreted pre-FadA consisting of 129 amino acids and the other is a secreted mature FadA $(\mathrm{mFadA})$ consisting of 111 amino acids. Pre-FadA is anchored in the $F n$ membrane, and $\mathrm{mFadA}$ is exposed on the surface of the bacteria. Together, they form a high molecular weight complex that can attach to endothelial cadherin, which causes a relocation of cadherin away from the cell-cell junctions. This results in an endothelium so permeable that even bacteria can pass. ${ }^{24}$ High-risk PD pathogens can increase endothelial permeability via multiple mechanisms.

\section{PATHOGENESIS, PART 3: LIPOPROTEIN BINDING IN INTIMA}

The third requirement for the pathogenesis of ASVD is binding of the lipoproteins in the intimal layer of the arterial wall. Perfusion studies with labelled lipoproteins show most lipoproteins that penetrate the endothelium diffuse through all the layers and efflux on the adventitial side. The numbers of retained lipoproteins in the intima are several orders of magnitude less than the number of particles that diffuse through the arterial wall. ${ }^{25}$ Therefore, retention of lipoproteins in the intima is a vital step in the pathogenesis of ASVD. The type of lipoprotein captured in the wall is not as important as the quantity of lipoproteins retained. Binding occurs via electrostatic forces to proteoglycans found in the extracellular matrix (ECM) of the intima. Proteoglycans have negatively charged areas, which bind to positively charged areas of amino acids on ApoB. There are two sites of attachment on ApoB-100, with one of those exposed only in sd-LDL particles. There is one site of attachment on ApoB-48. ${ }^{26}$

Smooth muscle cells (SMCs) have two phenotypes. One is the contractile phenotype found in the medial layer of the arterial wall. The other is the synthetic phenotype that migrates from the medial layer to the intimal layer. Synthetic SMCs create a proteoglycan-enriched ECM in the intima. ${ }^{27}$ Studies demonstrate that synthetic SMCs are the first intimal cells present in locations destined to develop ASVD. ${ }^{28}$ Microbial infection is known to stimulate genetic transcription of contractile SMCs to synthetic SMCs. ${ }^{29}$ PD due to the high-risk pathogen $\mathrm{Pg}$ has been shown to influence the levels of angiopoietin 1 (Angpt1) and angiopoietin 2 (Angpt2) in SMCs. P. gingivitis creates higher levels of Angpt2, which stimulates a genetic transformation of SMCs to the synthetic phenotype. Zhang et $a l^{30}$ 
concluded this is another mechanism by which periodontitis is associated with ASVD. PD has the potential to increase the binding of lipoproteins in the intima by enhancing the quantity of proteoglycans in intimal ECM.

\section{DISCUSSION}

PD due to high-risk pathogens may facilitate the three critical steps in the pathogenesis of ASVD (figure 1). Therefore, it is reasonable that $\mathrm{PD}$, due to the high-risk pathogens $(\mathrm{Aa}, \mathrm{Pg}, \mathrm{Td}$, $T f, F n$ ), be considered causal of ASVD on clinical grounds. Since ASVD is a complex multifactorial disease process, PD due to high-risk pathogens is a contributory cause. This means such $\mathrm{PD}$ is neither required nor sufficient for the pathogenesis of ASVD. ${ }^{7}$ It is necessary to elevate the distinction of PD to causal, as opposed to simply associated, for clinical management purposes. Causal classification requires therapy to mitigate the risk of its effect. In this case, it means PD due to these high-risk microbes must be treated effectively to reduce the risk of ASVD.

As is often the case in science, a new realisation creates a list of unsolved issues. In this case, one obvious question is how to objectively identify the pathogens. The definition of PD must include a diagnosis of the specific underlying pathogens causing the PD. There are numerous studies that have demonstrated the
CV risk from PD is driven by the pathogen burden as opposed to only clinical exam findings such as pocket depth, bleeding on probing and bone loss. ${ }^{31-33}$ The clinical oral health examination is important and must remain an important component of the diagnosis. However, from a CV standpoint, there must be an objective assessment of high-risk pathogen burden as part of the definition of PD. There are several DNA-based oral pathogen tests available to assess high-risk pathogens. However, many dentists are not familiar with these tests, and there is a substantial cost involved. Understanding that high-risk PD pathogens are causal of ASVD places an onus upon our dental colleagues to continue their efforts to develop affordable, reproducible, objective testing for high-risk PD pathogens.

An additional substantial issue following the recognition of PD due to high-risk pathogens as causal of ASVD is: how is it successfully managed? There is sparse data addressing the most effective way to manage such disease. One randomised prospective study of 101 patients with PD tested the effectiveness of scaling and root planning (SRP) alone versus SRP plus systemic antibiotics. There were two antibiotic arms: metronidazole (MTZ) $400 \mathrm{mg}$ three times per day or MTZ+ amoxicillin (AMX) (500 mg three times per day) for 14 days. In addition, half of the patients in each of the three arms of therapy rinsed

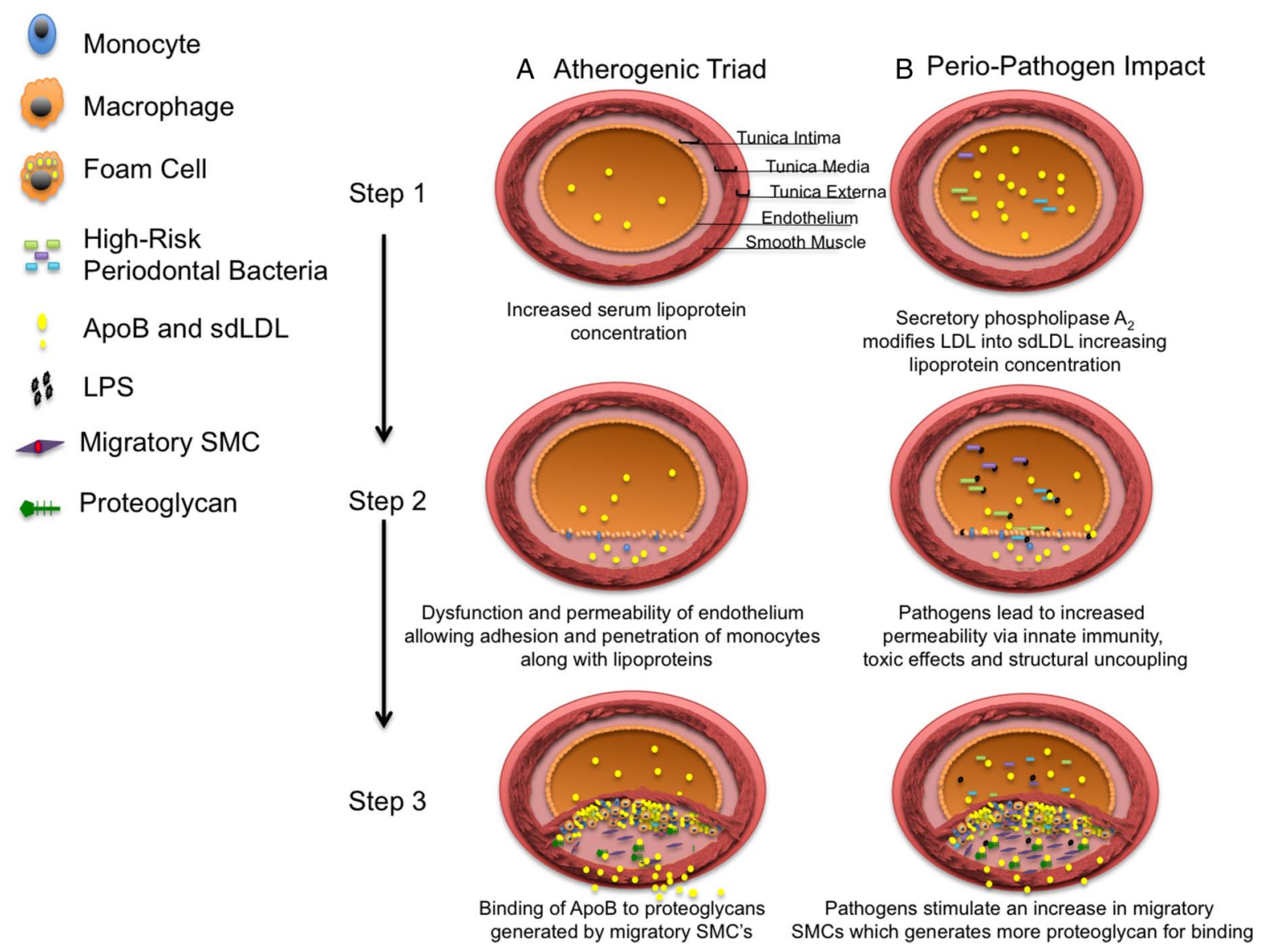

Figure 1 Atherogenic triad in the presence and absence of high-risk periodontal pathogens. Column A: Illustration of the atherogenic triad. (1) Lipoprotein concentration in the serum, with each lipoprotein containing ApoB. (2) Dysfunction and permeability of the endothelium which allow monocytes to adhere to the endothelium and to penetrate into the intima along with lipoproteins. (3) Binding of ApoB to proteoglycans derived from migratory SMCs, as well as conversion of monocytes to macrophages, which become foam cells. Column B: Illustration of how high-risk periodontal pathogens adversely enhance each of the three elements causing greater atherogenesis. (1) In the presence of high-risk pathogens, the concentration of $A p o B$ is increased. (2) In the presence of high-risk pathogens, the endothelial dysfunction and permeability are enhanced by LPS. (3) In the presence of high-risk pathogens, the binding of lipoproteins is enhanced by increased migratory SMCs, which enrich the intima with proteoglycans. ApoB, apolipoprotein B; LDL, low-density lipoprotein; LPS, lipopolysaccharides; sdLDL, small dense LDL; SMCs, smooth muscle cells. 
with $0.12 \%$ chlorhexidine two times per day for 2 months. Follow-up testing of pathogens was concluded at 1 year. The study found there was more effective elimination of high-risk pathogens with the addition of antibiotics and antimicrobial rinse. However, they point out the study might have been underpowered and any conclusions should be interpreted with caution. $^{34}$ One other randomised placebo-controlled clinical trial has been done comparing the effectiveness of SRP alone with SRP with the antibiotics MTZ and AMX. This study examined 58 non-smoking type 2 diabetics. They were treated with SRP alone or with MTZ $400 \mathrm{mg}$ and AMX $500 \mathrm{mg}$ three times per day for 14 days. Follow-up DNA evaluation for high-risk pathogens was concluded in 1 year. The patients treated with antibiotics had greater reduction in three high-risk pathogens: $T f, T d, P g$. Again, this is a very small study, and results need to be interpreted with caution. ${ }^{35}$ Issues with management extend beyond simply the initial therapy of high-risk pathogens. What is the most effective manner in which to maintain periodontal health? There is no significant published study, we are aware of, that addresses this pertinent issue. Studies evaluating management would also need to clarify the percentage of success, the side effects of therapy, the cost of the management and ultimately the return on investment in reducing the mortality and morbidity of ASVD. Currently, there are no large clinical trials generating a definitive answer for the effective management of PD due to high-risk pathogens. This is fertile research ground for our dental colleagues. Realising PD due to high-risk pathogens as causal of ASVD demands such investigation.

It should be clear that one reason the AHA's meta-analysis was only able to prove an independent association of PD to ASVD as opposed to a causal relationship is due to the paucity of studies including pathogen burden in the definition of PD. ${ }^{5}$ This remains a significant issue, as the studies currently published analyse the relationship between PD and ASVD. ${ }^{36-38}$ When the component of high-risk pathogens is included in the diagnosis of PD, there is evidence that treatment reduces $\mathrm{CV}$ risk. The carotid intimal media thickening (cIMT) change is a marker of change in CV risk. Treatment of high-risk pathogens effected cIMT in a positive manner. ${ }^{39}$ With the current scientific evidence that it is the high-risk PD pathogens which drive the $\mathrm{CV}$ risk, it is imperative that study protocols include specific pathogen burden as an inherent element in the definition of PD and that the therapeutic impact examines the change in highrisk pathogen burden. One would anticipate the results of such studies would be compatible with high-risk pathogens being a contributory cause of ASVD.

ASVD remains the number one cause of mortality and morbidity in this country. ${ }^{40}$ Current literature supports that it is more cost-effective to prevent ASVD than to treat its complications. ${ }^{41}$ Arterial disease has a common core cause, which is inflammation. ${ }^{42}$ There are a multitude of conditions that can generate arterial inflammation. The list includes: lipids, smoking, hypertension, insulin resistance, vitamin D deficiency, obstructive sleep apnoea, obesity, diet, physical inactivity, psychosocial issues, oral health issues, systemic inflammatory conditions such as rheumatoid arthritis, lupus, genetic influences, and systemic infectious disease and gut dysbiosis. ${ }^{43-57}$ We now have evidence that PD can cause systemic inflammation and can also adversely affect the three essential mechanisms in the pathogenesis of ASVD. It is necessary to classify PD due to high-risk pathogens as a cause of ASVD. This type of PD is a medical condition with a dental solution. At present, our dental colleagues have not elucidated a definitive way in which to successfully manage this type of PD. The pathway is clear for justifying that PD due to high-risk pathogens is causal of ASVD. Once our healthcare partners in dentistry have identified effective management of this type of PD, such intervention offers significant potential for reducing the impact of ASVD. ${ }^{58}$

\section{Main messages}

- Periodontal disease (PD) due to high-risk pathogens can adversely influence the atherosclerosis pathogenesis triad.

- PD caused by high-risk pathogens may be considered a contributory cause of arterial disease.

- The dental community has a substantial opportunity to mitigate the number one cause of morbidity and mortality, namely cardiovascular disease, by elucidating feasible effective management of PD due to high-risk pathogens.

\section{Current research questions}

This review propels the need for prospective trials, which would investigate the following inquiries:

- Prospective randomised trials are needed to define effective management of periodontal disease (PD) due to high-risk pathogens. These studies must also address the cost of such management along with the side effects, long-term effectiveness and benefit in terms of mitigating CV events.

- In patients with PD, does successful eradication of high-risk pathogens lower apolipoprotein B levels?

- Utilising labelled lipoproteins in an arterial wall perfusion study, is the quantity of lipoprotein intimal retention different before and after therapy for high risk PD pathogens?

\section{Key references}

- Cobe HM. Transitory bacteremia. Oral Surg Oral Med Oral Pathol 1954;7:609-15.

- Figuero E, Sánchez-Beltrán $M$, Cuesta-Frechoso $S$, et al. Detection of periodontal bacteria in atheromatous plaque by nested polymerase chain reaction. $J$ Periodontol 2011;82:1469-77.

- Gaetti-Jardim E Jr, Marcelino SL, Feitosa AC, et al. Quantitative detection of periodontopathic bacteria in atherosclerotic plaques from coronary arteries. $\mathrm{J} \mathrm{Med}$ Microbiol 2009;58(Pt 12):1568-75.

- Li X, Kolltveit KM, Tronstad L, et al. Systemic diseases caused by oral infection. Clin Microbiol Rev 2000;13:54758.

- Zhang B, Khalaf H, Sirsjo A, et al. Gingipains from the periodontal pathogen Porphyromonas gingivalis play a significant role in regulation of angiopoietin 1 and angiopoietin 2 in human aortic smooth muscle cells. Infect Immun 2015;83:4256-65. 


\section{Self assessment questions (True/False)}

1. Level A evidence demonstrates that periodontal disease is independently associated with atherosclerotic vascular disease.

2. A contributory cause does not require that all those who possess the contributory cause experience the effect, nor does it require that all those who are free of the contributory cause be free of the effect.

3. Periodontal disease due to high-risk pathogens can play a direct role in the triad of conditions influencing the pathogenesis of atherosclerosis.

4. High-risk periodontal pathogens cannot influence lipoprotein serum concentration or endothelial permeability.

5. The high-risk periodontal pathogen Porphyromonas gingivalis can promote the conversion of contractile smooth muscle cells into migratory synthetic smooth muscle cells, which can enhance the binding of lipoproteins in the intima.

\section{Competing interests None declared.}

Provenance and peer review Not commissioned; externally peer reviewed.

Open Access This is an Open Access article distributed in accordance with the Creative Commons Attribution Non Commercial (CC BY-NC 4.0) license, which permits others to distribute, remix, adapt, build upon this work non-commercially, and license their derivative works on different terms, provided the original work is properly cited and the use is non-commercial. See: http://creativecommons.org/ licenses/by-nc/4.0/

\section{REFERENCES}

1 Cobe HM. Transitory bacteremia. Oral Surg Oral Med Oral Pathol 1954;7:609-15.

2 Li X, Kolltveit KM, Tronstad L, et al. Systemic diseases caused by oral infection. Clin Microbiol Rev 2000;13:547-58.

3 Gaetti-Jardim E Jr, Marcelino SL, Feitosa AC, et al. Quantitative detection of periodontopathic bacteria in atherosclerotic plaques from coronary arteries. J Med Microbiol 2009;58(Pt 12):1568-75.

4 Figuero E, Sánchez-Beltrán $M$, Cuesta-Frechoso $S$, et al. Detection of periodontal bacteria in atheromatous plaque by nested polymerase chain reaction. J Periodontol 2011;82:1469-77.

5 Lockhart PB, Bolger AF, Papapanou PN, et al. Periodontal disease and atherosclerotic vascular disease: does the evidence support an independent association?: a scientific statement from the American Heart Association. Circulation 2012; 125:2520-44

6 Wilkins JT, Li RC, Sniderman A, et al. Discordance between apolipoprotein B and LDL-cholesterol in young adults predicts coronary artery calcification: the CARDIA study. I Am Coll Cardiol 2016;67:193-201.

7 Riegelman R. Contributory cause: unnecessary and insufficient. Postgrad Med 1979;66:177-9.

8 Rosenson RS, Hegele RA, Gotto AM Jr. Integrated measure for atherogenic lipoproteins in the modern era: risk assessment based on apolipoprotein B. J Am Coll Cardiol 2016:67:202-4.

9 Gotto AM, Jr, Whitney E, Stein EA, et al. Relation between baseline and on-treatment lipid parameters and first acute major coronary events in the Air Force/ Texas Coronary Atherosclerosis Prevention Study (AFCAPS/TexCAPS). Circulation 2000;101:477-84.

10 Thanassoulis G, Williams K, Ye K, et al. Relations of change in plasma levels of LDL-C, non-HDL-C and apoB with risk reduction from statin therapy: a meta-analysis of randomized trials. J Am Heart Assoc 2014:3:e000759.

11 Hayashi T, Hirano T, Shiobara T, et al. [Small dense LDL concentration is closely associated with serum apolipoprotein B, comparisons of non-LDL cholesterol or LDL cholesterol]. Rinsho Byori 2006;54:569-75.

12 Sartipy P, Camejo G, Svensson L, et al. Phospholipase A2 modification of lipoproteins: potential effects on atherogenesis. Adv Exp Med Biol 2002;507:3-7.

13 Boillot A, Demmer RT, Mallat Z, et al. Periodontal microbiota and phospholipases: the Oral Infections and Vascular Disease Epidemiology Study (INVEST). Atherosclerosis 2015;242:418-23.
14 Parameter on aggressive periodontitis. American Academy of Periodontology. J Periodontol 2000;71(5 Suppl):867-9.

15 Rufail ML, Schenkein HA, Barbour SE, et al. Altered lipoprotein subclass distribution and PAF-AH activity in subjects with generalized aggressive periodontitis. J Lipid Res 2005;46:2752-60

16 Wang H, Patterson C. Atherosclerosis: risks, mechanisms, and therapies. Hoboken, New Jersey: Wiley Blackwell, 2015.

17 Vanhoutte PM. Endothelial dysfunction: the first step toward coronary arteriosclerosis. Circ J 2009;73:595-601.

18 Mann DL. The emerging role of innate immunity in the heart and vascular system: for whom the cell tolls. Circ Res 2011;108:1133-45.

19 Galkina E, Ley K. Vascular adhesion molecules in atherosclerosis. Arterioscler Thromb Vasc Biol 2007:27:2292-301.

20 McKenzie JA, Ridley AJ. Roles of Rho/ROCK and MLCK in TNF-alpha-induced changes in endothelial morphology and permeability. I Cell Physiol 2007;213:221-8

21 Deanfield JE, Halcox JP, Rabelink TJ. Endothelial function and dysfunction: testing and clinical relevance. Circulation 2007;115:1285-95.

22 Dietmann A, Millonig A, Combes V, et al. Effects of Aggregatibacter actinomycetemcomitans leukotoxin on endothelial cells. Microb Pathog 2013;6162:43-50.

23 Harris ES, Nelson WJ. VE-cadherin: at the front, center, and sides of endothelial cell organization and function. Curr Opin Cell Biol 2010;22:651-8.

24 Fardini $Y$, Wang $X$, Témoin $S$, et al. Fusobacterium nucleatum adhesin FadA binds vascular endothelial cadherin and alters endothelial integrity. Mol Microbiol 2011:82:1468-80.

25 Proctor SD, Mamo JC. Intimal retention of cholesterol derived from apolipoprotein B100- and apolipoprotein B48-containing lipoproteins in carotid arteries of Watanabe heritable hyperlipidemic rabbits. Arterioscler Thromb Vasc Biol 2003;23:1595-600.

26 Fogelstrand P, Borén J. Retention of atherogenic lipoproteins in the artery wall and its role in atherogenesis. Nutr Metab Cardiovasc Dis 2012:22:1-7.

27 Rudijanto $A$. The role of vascular smooth muscle cells on the pathogenesis of atherosclerosis. Acta Med Indones 2007;39:86-93.

28 Doran AC, Meller N, McNamara CA. Role of smooth muscle cells in the initiation and early progression of atherosclerosis. Arterioscler Thromb Vasc Biol 2008:28:812-19.

29 Libby P, Hansson GK. Inflammation and immunity in diseases of the arterial tree: players and layers. Circ Res 2015:116:307-11.

30 Zhang $B$, Khalaf $H$, Sirsjö $A$, et al. Gingipains from the periodontal pathogen Porphyromonas gingivalis play a significant role in regulation of angiopoietin 1 and angiopoietin 2 in human aortic smooth muscle cells. Infect Immun 2015;83:4256-65.

31 Desvarieux M, Demmer RT, Jacobs DR Jr, et al. Periodontal bacteria and hypertension: the oral infections and vascular disease epidemiology study (INVEST). $J$ Hypertens 2010;28:1413-21.

32 Demmer RT, Desvarieux M. Periodontal infections and cardiovascular disease: the heart of the matter. J Am Dent Assoc 2006;137:14S-20S; quiz 38S.

33 Desvarieux M, Demmer RT, Rundek T, et al. Periodontal microbiota and carotid intima-media thickness: the Oral Infections and Vascular Disease Epidemiology Study (INVEST). Circulation 2005;111:576-82.

34 Soares GM, Mendes JA, Silva MP, et al. Metronidazole alone or with amoxicillin as adjuncts to non-surgical treatment of chronic periodontitis: a secondary analysis of microbiological results from a randomized clinical trial. I Clin Periodontol 2014:41:366-76.

35 Miranda TS, Feres M, Perez-Chaparro PJ, et al. Metronidazole and amoxicillin as adjuncts to scaling and root planing for the treatment of type 2 diabetic subjects with periodontitis: 1-year outcomes of a randomized placebo-controlled clinical trial. J Clin Periodontol 2014:41:890-9.

36 Marfil-Álvarez R, Mesa F, Arrebola-Moreno A, et al. Acute myocardial infarct size is related to periodontitis extent and severity. J Dent Res 2014:93:993-8.

$37 \mathrm{Yu}$ YH, Chasman DI, Buring JE, et al. Cardiovascular risks associated with incident and prevalent periodontal disease. J Clin Periodontol 2015;42:21-8.

38 Rydén $\mathrm{L}$, Buhlin $\mathrm{K}$, Ekstrand $\mathrm{E}$, et al. Periodontitis increases the risk of a first myocardial infarction: a report from the PAROKRANK study. Circulation 2016:133:576-83.

39 Desvarieux M, Demmer RT, Jacobs DR, et al. Changes in clinical and microbiological periodontal profiles relate to progression of carotid intima-media thickness: the Oral Infections and Vascular Disease Epidemiology study. J Am Heart Assoc 2013;2: e000254.

40 Mozaffarian D, Benjamin EJ, Go AS, et al. Heart disease and stroke statistics2015 update: a report from the American Heart Association. Circulation 2015;131: e29-322.

41 Baber U, Mehran R, Sartori S, et al. Prevalence, impact, and predictive value of detecting subclinical coronary and carotid atherosclerosis in asymptomatic adults: the Biolmage study. J Am Coll Cardiol 2015;65:1065-74.

42 Swerdlow DI, Holmes MV, Kuchenbaecker KB, et al., Interleukin-6 Receptor Mendelian Randomisation Analysis Consortium. The interleukin-6 receptor as a 
target for prevention of coronary heart disease: a mendelian randomisation analysis. Lancet 2012;379:1214-24.

43 Toutouzas K, Benetos G, Drakopoulou M, et al. Incremental predictive value of carotid inflammation in acute ischemic stroke. Stroke 2015:46:272-4.

44 Weinberg EO, Genco CA. Directing TRAF-ic: cell-specific TRAF6 signaling in chronic inflammation and atherosclerosis. Circulation 2012;126:1678-80.

45 Csiszar A, Podlutsky A, Wolin MS, et al. Oxidative stress and accelerated vascular aging: implications for cigarette smoking. Front Biosci (Landmark Ed) 2009;14:3128-44.

46 Boos CJ, Lip GY. Elevated high-sensitive C-reactive protein, large arterial stiffness and atherosclerosis: a relationship between inflammation and hypertension? I Hum Hypertens 2005;19:511-13.

47 Kim TN, Kim S, Yang SJ, et al. Vascular inflammation in patients with impaired glucose tolerance and type 2 diabetes: analysis with 18F-fluorodeoxyglucose positron emission tomography. Circ Cardiovasc Imaging 2010;3:142-8.

48 Lavie CJ, Lee JH, Milani RV. Vitamin D and cardiovascular disease will it live up to its hype? J Am Coll Cardiol 2011;58:1547-56.

49 Jelic S, Lederer DJ, Adams T, et al. Vascular inflammation in obesity and sleep apnea. Circulation 2010;121:1014-21.

50 Keaney JF Jr, Larson MG, Vasan RS, et al. Obesity and systemic oxidative stress: clinical correlates of oxidative stress in the Framingham Study. Arterioscler Thromb Vasc Biol 2003;23:434-9.

51 Tang WH, Wang Z, Levison BS, et al. Intestinal microbial metabolism of phosphatidylcholine and cardiovascular risk. N Engl J Med 2013;368:1575-84.

52 Mora S, Cook N, Buring JE, et al. Physical activity and reduced risk of cardiovascular events: potential mediating mechanisms. Circulation 2007:116:2110-18.

53 Steptoe A, Molloy GJ, Messerli-Bürgy N, et al. Fear of dying and inflammation following acute coronary syndrome. Eur Heart I 2011;32:2405-11.
54 Fifer KM, Qadir S, Subramanian S, et al. Positron emission tomography measurement of periodontal $18 \mathrm{~F}$-fluorodeoxyglucose uptake is associated with histologically determined carotid plaque inflammation. J Am Coll Cardiol 2011:57:971-6.

55 Fischer LM, Schlienger RG, Matter C, et al. Effect of rheumatoid arthritis or systemic lupus erythematosus on the risk of first-time acute myocardial infarction. $\mathrm{Am}$ J Cardiol 2004;93:198-200.

56 Harismendy 0 , Notani $D$, Song $X$, et al. 9p21 DNA variants associated with coronary artery disease impair interferon- $\gamma$ signalling response. Nature 2011;470:264-8

57 Dalager-Pedersen M, Søgaard M, Schønheyder HC, et al. Risk for myocardial infarction and stroke after community-acquired bacteremia: a 20-year population-based cohort study. Circulation 2014;129:1387-96

58 Eke PI, Dye BA, Wei L, et al. Prevalence of periodontitis in adults in the United States: 2009 and 2010. J Dent Res 2012;91:914-20.

\section{Answers}

1. True

2. True

3. True

4. False

5. True 